\title{
Acknowledgement to Reviewers of Insects in 2018
}

\author{
Insects Editorial Office \\ MDPI, St. Alban-Anlage 66, 4052 Basel, Switzerland \\ Published: 9 January 2019
}

Rigorous peer-review is the corner-stone of high-quality academic publishing. The editorial team greatly appreciates the reviewers who contributed their knowledge and expertise to the journal's editorial process over the past 12 months. In 2018, a total of 196 papers were published in the journal, with a median time to first decision of 21 days and a median time to publication of 48 days. The editors would like to express their sincere gratitude to the following reviewers for their cooperation and dedication in 2018:

Abro, G. H.

Adang, Michael

Addesso, Karla

Adelman, Zachary

Ahn, Jeongjoon

Akhtar, Yasmin

Alburaki, Mohamed

Alford, Lucy

Allen, Margaret L.

Alout, Haoues

Amiri, Esmaeil

Anderbrant, Olle

Andersen, Jeremy C.

Anton, Sylvia

Appel, Arthur G.

Arthurs, Steven

Athanassiou, Christos

Augustinos, Antonios

Avtzis, Dimitrios N.

Ayayee, Paul

Baker, Melissa

Ballardini, Marco

Balzan, Stefania

Barber-James, Helen M.

Barman, Apurba

Battisti, Andreas

Baxter, Simon

Beaurepaire, Alexis

Beebe, Nigel

Benecke, Mark

Benedict, Mark Q.

Bennett, Meghan M.

Berenbaum, May R.

Bernardo, Umberto

Beyenbach, Klause W.
Bierzychudek, Paulette

Bignell, David

Bjørge, Julie

Black, William C.

Blacquière, Tjeerd

Blair, Carol

Bocak, Ladislav

Bohman, Björn

Bonali, Fabio Luca

Bonilla-Rosso, Germán

Borges, Paulo A. V.

Bose, Joy

Bosso, Luciano

Brackney, Doug

Bradshaw, Terence

Brambila, Julieta

Brankatschk, Marko

Breeuwer, Johannes A. J.

Brent, Colin

Britch, S. C.

Broughton, Sonya

Brownbridge, Michael

Brückner, Adrian

Brundage, Adrienne

Brunet, Bryan M. T.

Brutscher, Laura $\mathrm{M}$.

Bryant, William B.

Buchanan, Amanda

Buitenhuis, Rose

Burgio, Giovanni

Burkett-Cadena, Nathan

Burrows, Andrea

Calkins, Travis

Campbell, Ewan

Campolo, Orlando 


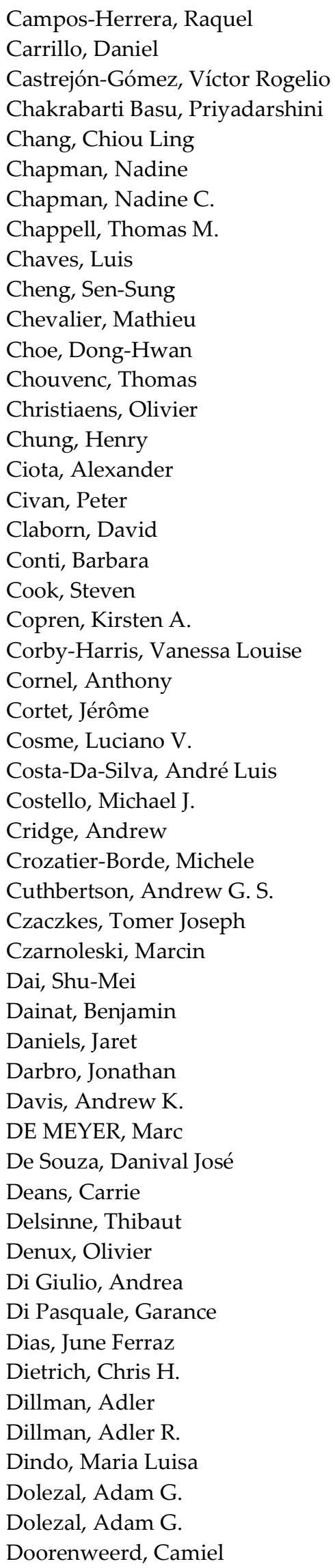

\author{
Doremus, Matthew \\ Dorin, Alan \\ Doube, Bernard \\ Dowling, Andrea \\ Droege, Sam \\ Duan, Jian \\ Dupuis, Julian \\ Duso, Carlo \\ Dyer, Adrian \\ Dyson, Paul J. \\ Eady, Paul \\ Eliopoulos, Panagiotis A. \\ Elkinton, Joe \\ Endersby, Nancy Margaret \\ Epsky, Nancy D. \\ Erram, Dinesh \\ Fahrbach, Susan \\ Feldhaar, Heike Feldhaar \\ Fellowes, Mark \\ Fleming, Daniel \\ Flenniken, Michelle \\ Forister, Matt \\ Fornoff, Felix \\ Foster, Woodbridge \\ Foy, Brian \\ Frank, Erik T. \\ Frank, Kevin \\ Freund, Friedemann \\ Fritz, Megan L. \\ Funderburk, Joe \\ G S Cuthbertson, Andrew \\ Garcia, Flávio Roberto Mello \\ Gendrin, Mathilde \\ Gezon, Zak \\ Ghosh, Subir \\ Gibbs, Jason \\ Gile, Gillian \\ Gill, Harsimran K. \\ Giovenazzo, Pierre \\ Gisder, Sebastian \\ Giunti, Giulia \\ Gloag, Ros \\ Goff, Gaëlle Le \\ Golick, Doug \\ Gomez-Marco, Francesc \\ Gondhalekar, Ameya D. \\ González-Zamora, Jose E \\ Gotoh, Hiroki \\ Grabenweger, Giselher \\ Grace, Kenneth \\ Grassl, Julia \\ Greenslade, Penelope
}


Gregg, Peter

Griffin, Christine

Grof-Tisza, Patrick

Gross, Aaron

Großhans, Jörg

Gruner, Susan

Gruwell, Matthew

Grzywacz, Andrzej

Guastella, Devid

Guerra, Patrick Anthony

Guerrero, Angel

Guis, Hélène

Haddi, Khalid

Haelewaters, Danny

Halbert, Susan

Hall, David

Hallem, Elissa A.

Hamby, Kelly

Hanly, Joseph J.

Hansen, Allison

Harbison, Justin

Hart, Adam

Hassan, Errol

Hatt, Séverin

Healy, Kristen

Heerman, Matthew

Hemerik, Lia

Hendriksma, Harmen

Heterick, Brian E.

Hill, Geena

Hill, Matt

Hillyer, Julián F.

Hoffmann, Christoph

Hoffmann, Klaus

Hogsette, Jerome

Holden, Matthew $\mathrm{H}$.

Hope, Sandra

Horgan, Finbarr G.

Hottel, Benjamin

Howlett, Brad

Hribar, Lawrence

$\mathrm{Hu}, \mathrm{Yi}$

Huang, Xin

Huang, Yan-Jang

Huang, Zachary

Hutchison, William

Ibrahim, Sulaiman Sadi

Ingram, Erin

Isman, Murray B.

Jacobsen, Stine Kramer

Jaeger, Henry

Jaenike, John
Jaffe, Benjamin

Johnson, Kelly S.

Johnson, Reed

Johnston, J. Spencer

Jones, Carol

Jones, David

Jones, David T.

Jones, Julia

Joseph, Shimat

Joshi, Neelendra K.

Jouquet, Pascal

Judd, Timothy

Jung, Chuleui

Jürgens, Andreas

Kageyama, Daisuke

Kainoh, Yooichi

Kalsi, Megha

Kang, Seokyoung

Karamaouna, Filitsa

Karban, Richard

Kawasaki, Hideki

Keena, Melody

Kehrli, Patrik

Khadempour, Lily

Kianifariz, Mahnaz

Kim, Dohyeong

Kirker, Grant

Kiuchi, Takashi

Kleier, Daniel A.

Klingen, Ingeborg

Knight, Alan

Knisley, C. Barry

Kohler, Steve

Konagaya, Tatsuro

Kooij, Pepijn W.

Koou, Sin-Ying

Koyama, Takashi

Kozlov, Mikhail

Krischik, Vera A.

Krishnan, Natraj

Kristensen, Torsten Nygård

Kumar, Pradeep

Kumar, Vivek

Kuo, Ping-Chung

Kupper, Maria

Kvamme, Torstein

Kwong, Waldan K.

Lahiri, Sriyanka

Lahondere, Chloe

Lamp, Benjamin

Lampert, Niclas

Lander, Tonya 
Lapointe, Stephen

Lavista Llanos, Sofía

Lawler, Sharon

Lazzari, Claudio

Leather, Simon

Lecocq, Antoine

Lécureuil, Charlotte

Lee, Jana C.

Lee, Joon-hak

Lees, Rosemary Susan

Lehmann, Philipp

Lester, P. J.

Liao, Ling-Hsiu

Linnakoski, Riikka

Lobo, Neil

Loni, Augusto

Looney, Chris

Lopez-Ferber, Miguel

López-López, Alejandro

Luckhart, Shirley

Lundin, Ola

Ma, Guojia

Macias, Vanessa M.

MacLean, Heidi J.

Magura, Tibor

Mainali, Bishwo

Maistrello, Lara

Malacrinò, Antonino

Malcolm, Stephen

Mandrioli, Mauro

Manfredini, Fabio

Manino, Aulo

Mankin, Richard W.

Mann, Rajinder S.

Mantilla-Contreras, Jasmin

Mantzoukas, Spiridon

Marchant, Richard

Marini, Mario

Marshall, Katie E.

Martel, Véronique

Martin, Arnaud

Martin, Stephen

Martínez, Luis Carlos

Martins, Fabricio

Martín-Vega, Daniel

Massimino Cocuzza, Giuseppe

Mattei, Cesar

Maynard, Daniel

Mazzoni, Emanuele

McCravy, Kenneth W.

McDonald, Allison

McEvoy, Peter
McFarland, Kent

McNeil, Jeremy N.

McNeill, Corraine

Meiklejohn, Kelly A.

Merchant, Michael

Meuti, Megan

Michalik, Anna

Milbrath, Meghan O.Grady

Miles, Carol I.

Millán, Andrés

Miller, Wolfgang

Milotic, Tanja

Mitchell, Forrest L.

Mlocicki, Daniel

Moar, William J.

Mody, Karsten

Mogilicherla, Kanakachari

Mogren, Christina

Mooney, Emily H.

Morales-Ramos, Juan A.

Morandin, Claire

Moreno, Aránzazu

Mori, Boyd

Moriyama, Minoru

Morrison, William R.

Morse, Douglass H.

Mound, Laurence A.

Moura, Luciano De Azevedo

Mullins, Aaron J.

Murchie, Archie K.

Murillo, Rosa

Mutinelli, Franco

Nagaoka, Sumiharu

Narouei-Khandan, Hossein A.

Navarro, Shlomo

Nedvěd, Oldřich

Neumann, Peter

Neupane, Avishesh

Nielsen, Anne

Nieslen, Anne

Niño, Elina

Nishiwaki, Hisashi

Nogami, Makoto

Noriyuki, Suzuki

Nottingham, Louis

Nugnes, Francesco

Numata, Hideharu

Nuss, Andrew B.

Oi, David H.

Oi, Faith

Okada, Kensuke

O'Neal, Scott 
Oppenheim, Sara

Ortiz-Urquiza, Almudena

Osada, Yohei

Osbrink, Weste L.

Ostiguy, Nancy

Otuka, Akira

Overgaard, Johannes

Ozaki, Katsuhisa

Paploski, Igor

Pappas, Maria L.

Park, Hyun-Woo

Park, Il-Kwon

Park, Kye Chung

Park, Soo Jean

Patrick, Marjorie

Pavela, Roman

Pearson, Gwen

Perera, Omaththage

Peterson, Brittany F.

Pettersson, Lars

Pfeiffer, Douglas

Picimbon, Jean-François

Pickett, Charles H.

Pirk, Christian

Pleasants, John M

Pondeville, Emilie

Pope, Karen L.

Popham, Holly J.

Portilla, Maribel

Poudel, Barun

Prasad, Abhishek

Querner, Pascal

Rand, Tatyana A.

Rangel, Juliana

Ranger, Christopher

Ravenscraft, Alison

Razze, Janine

Reddy, Gadi

Rehman, Junaid U.

Rendon, Dalila

Renkema, Justin

Renkema, Justin M.

Ribeiro, José

Ricupero, Michele

Riddick, Eric

Riehle, Michael

Rijal, Jhalendra

Riley, David G.

Rinkevich, Frank

Robinson, Gene E.

Roditakis, Emmanouil

Rodriguero, Marcela
Rodríguez-Rojas, Alexandro

Roggero, Angela

Romero, Alvaro

Rondoni, Gabriele

Roubik, David W.

Roubos, Craig R

Rouhier, Matthew F.

Roy, Sourav

Ruberson, John R.

Ruiu, Luca

Rust, Michael

Ryabov, Eugene

Rybska, Eliza

Sagili, Ramesh

Saigusa, Kiyoshi

Salis, Lucia

Salom, Scott

Saloña-Bordas, Marta I.

San Jose, Michael

Sánchez-Bayo, Francisco

Sans, Albert

Santoiemma, Giacomo

Sato, Yukita

Savvides, Andreas

Sbordoni, Valerio

Scharf, Michael

Scheffrahn, Rudolf H.

Schetelig, Marc F.

Scholtens, Brian G.

Seal, Dakshina R.

Setamou, Mamoudou

Severns, Paul M.

Seymoure, Brett M.

Shapiro, Arthur

Shaw, Dana K.

Shearer, Peter W.

Shelly, Todd

Shi, Yi-Ming

Shockley, Marianne

Shostak, Allen

Shrestha, Mani

Sial, Ashfaq Ahmad

Siderhurst, Matthew

Siegel, Joel

Silk, Peter

Sim, Sheina

Simoes, Zila

Skelley, Paul E.

Skorokhod, Oleksii

Skovgård, Henrik

Slipinski, Adam

Smart, Matthew 
Smith, Hugh A.

Smith, Sarah M.

Smodiš Škerl, Maja

Snow, Janathan

Solensky, Michelle

Sparks, Michael

Spencer, Joseph L.

Spiteller, Dieter

Sponsler, Douglas

Srinivasan, Ramasamy

Stark, John D.

Sterzyńska, Maria

Stevens, Michael T.

Stockan, Jenni

Stockton, Dara

Storer, Nicholas P.

Stout, Michael

Strand, Michael R.

Strong, Donald R.

Studebaker, Glenn E.

Suh, Eunho

Suiter, Dan

Surapathrudu, Kanakala

Susset, Eline C.

Sweeney, Jon

Swengel, Ann B.

Switzer, Callin

Syed, Zainulabeuddin

Szumny, Antoni Jacek

Tabata, Jun

Tai, Helen

Tarasco, Eustachio

Tarasov, Sergei

Tatsuta, Haruki

Taylor, David

Tetreau, Guillaume

Thorn, Simon

Throne, James

Todd, Jacqui H.

Tojo, Koji

Tonkyn, Dave

Tosi, Simone

Toussaint, Emmanuel F.A.

Traver, Brenna

Trdan, Stanislav

Trematerra, Pasquale

Trotter, Talbot

Tschinkel, Walter R.

Tucker, Erika

Turell, Michael J.

Tzin, Vered

Unbehend, Melanie
Urban, Julie

Valles, Steven

Van Belleghem, Steven

Van Der Kooi, Casper

Vander Meer, Robert

Vankosky, Meghan A.

Varloud, Marie

Velez, Ana

Verheggen, François

Verhulst, Niels

Verneau, Fabio

Villalobos, Ethel M.

Villaverde, Juan José

Vuts, Jozsef

Wada-Katsumata, Ayako

Wagler, Ronald

Wagner, Sven

Walgenbach, James

Walker, Gregory

Waller, Deborah

Walton, William E.

Watari, Yasuhiko

Webb, Cameron

Weeks, Faith

Wells, Carrie

Wells, Jeffrey

Weston, Paul

Wetterer, James K.

Whitehorn, Penelope

Wiegmann, Brian M.

Wiesner, Rudolf

Wiggins, Greg

Wilhelm, Gertha

Wilson, Houston

Wilson, Joseph S.

Wilson, Michael

Winder, Linton

Wink, Michael

Wise, John

Wone, Bernard

Woyke, Jerzy

Wright, Denis

Wright, Mark

Xu, Guang

Xue, Rui-De

Yagound, Boris

Yamanaka, Naoki

Yanagawa, Aya

Yaninek, Steve

Yao, Jianxiu

Yashiro, Toshihisa

Yee, Wee L. 
Yoshioka, Akira

Zhang, Qing-He
Zografou, Konstantina

Zolnerowich, Gregory

(C) 2019 by the author. Licensee MDPI, Basel, Switzerland. This article is an open access article distributed under the terms and conditions of the Creative Commons Attribution (CC BY) license (http://creativecommons.org/licenses/by/4.0/). 